\section{Tissue-specific RNAi reveals that WT1 expression in nurse cells controls germ cell survival and spermatogenesis}

Manjeet K. Rao, ${ }^{1}$ John Pham, ${ }^{1}$ J. Saadi Imam, ${ }^{1}$ James A. MacLean, ${ }^{1}$ Deepa Murali, ${ }^{2}$ Yasuhide Furuta, ${ }^{2}$ Amiya P. Sinha-Hikim, ${ }^{3}$ and Miles F. Wilkinson ${ }^{1,4}$

${ }^{1}$ Department of Immunology, ${ }^{2}$ Department of Biochemistry and Molecular Biology, The University of Texas M.D. Anderson Cancer Center, Houston, Texas 77030, USA; ${ }^{3}$ Division of Endocrinology, University of California at Los Angeles School of Medicine, Torrance, California 90502, USA

Using a novel tissue-specific RNA interference (RNAi) approach that mimics the principle by which naturally occurring microRNAs (miRNA) are made, we demonstrate that the Wilms' tumor 1 (WT1) transcription factor has an essential role in spermatogenesis. Mice depleted of WT1 in Sertoli nurse cells suffered from increased germ cell apoptosis, loss of adherens junctions, disregulation of adherence junction-associated genes, and impaired fertility. These effects were recapitulated in transgenic mice expressing a dominant-negative form of WT1 in Sertoli cells, demonstrating the validity of our RNAi approach. Our results indicate that the tumor suppressor WT1 promotes Sertoli cell-germ cell signaling events driving spermatogenesis.

Supplemental material is available at http://www.genesdev.org.

Received August 23, 2005; revised version accepted November 28, 2005.

WT1 (the Wilms' tumor 1 transcription factor) is a tumor-suppressor gene essential for normal embryonic development that when mutated causes multiple human syndromes, resulting in birth defects and cancer (Little et al. 1999; Park and Jameson 2005). WT1 expression persists after birth in Sertoli nurse cells in the testis (Armstrong et al. 1993), but the functional significance of this expression is not known, as WT1-null mice die during embryogenesis (Little et al. 1999; Park and Jameson 2005). To address its postnatal and adult function, we developed a novel RNA interference (RNAi) approach to knock down WT1 expression in a tissue-specific manner in vivo. The RNAi approaches used by most investigators rely on polymerase (Pol) III promoters because these promoters terminate at a precise site, allowing for short ( 22-nucleotide [nt]) small interfering RNAs (siRNAs) to be made. However, Pol III promoters are ubiquitously expressed, and thus they cannot be used directly for tis-

[Keywords: WT1; germ cell; spermatogenesis; RNAi; Sertoli cell; miRNA] ${ }^{4}$ Corresponding author.

E-MAIL mwilkins@mdanderson.org,FAX (713) 563-3357.

Article and publication are at http://www.genesdev.org/cgi/doi/10.1101/ gad1367806. sue-specific knockdowns. In contrast, Pol II promoters can provide tissue-specific expression, but because they cannot generate siRNAs directly, they have been used instead to express long double-stranded RNAs (dsRNAs) that are processed by the RNase III enzyme Dicer to generate siRNAs. While powerful, this dsRNA approach can only be used in oocytes and embryonic stem cells, as other cell types respond to long dsRNAs (greater than $\sim 50 \mathrm{nt}$ ) by eliciting an interferon response that causes severe side effects (Fedoriw et al. 2004).

To circumvent this problem, we developed a Pol IIbased RNAi approach that does not generate long dsRNAs and thus can potentially be used in any cell type. In our approach, Pol II transcribes a precursor RNA containing a stem-loop that is cleaved by the ubiquitous nuclear RNase III enzyme Drosha. Thus, our approach mimics the means by which naturally occurring microRNAs (miRNAs) are generated (Fig. 1A). This Drosha-based approach has been shown to cleave artificially generated stem-loops expressed from premRNAs in transfected cell lines (Zeng and Cullen 2004). Using this approach, we generated transgenic mice with reduced WT1 levels in Sertoli cells in vivo. These siRNA-WT1 mice suffered from increased germ cell apoptosis and a loss of the adherens junction complexes that form between Sertoli cells and germ cells. Transgenic mice expressing a dominant-negative (DN) WT1 molecule in Sertoli cells exhibited the same phenotypic effects and alterations in gene expression, demonstrating the validity of our RNAi approach and unambiguously demonstrating that WT1 expression in Sertoli cells is crucial for spermatogenesis. To our knowledge, this is the first postnatal or adult function definitively assigned to WT1.

\section{Results and Discussion}

Tissue-specific knockdown of WT1 in vivo

To express a siRNA specific for WT1 in postnatal and adult Sertoli cells, we used the proximal promoter $(\mathrm{Pp})$ from the mouse Pem (Rhox5) gene, the founding member of a newly discovered homeobox gene cluster on the $\mathrm{X}$ chromosome (MacLean et al. 2005). The Pp was optimal for our purposes, as it is induced in Sertoli cells at the beginning of spermatogenesis (day 8 postpartum) (Lindsey and Wilkinson 1996; Sutton et al. 1998), precisely when WT1 mRNA levels peak (Pelletier et al. 1991). For our RNAi approach, we used 0.6-kb 5'-flanking sequences from the $\mathrm{Pp}$ (Fig. 1B), as we previously found this is sufficient to restrict expression to only Sertoli cells in the testis and principal cells in the caput epididymis (Rao et al. 2002, 2003). Downstream of the Pp we introduced sequences encoding a simple two-exon precursor RNA containing an intronic hairpin loop, one strand of which has perfect complementarity with a region of WT1 mRNA (Supplementary Fig. S1A). The WT1 complementary sequences we chose were previously shown to strongly inhibit WT1 expression in transfected cells when expressed as a chemically synthesized siRNA (Davies et al. 2004). The two exons in our siRNA-WT1 transgene lack ATG codons; therefore, the mature mRNA generated after splicing is not translatable and hence is innocuous. 

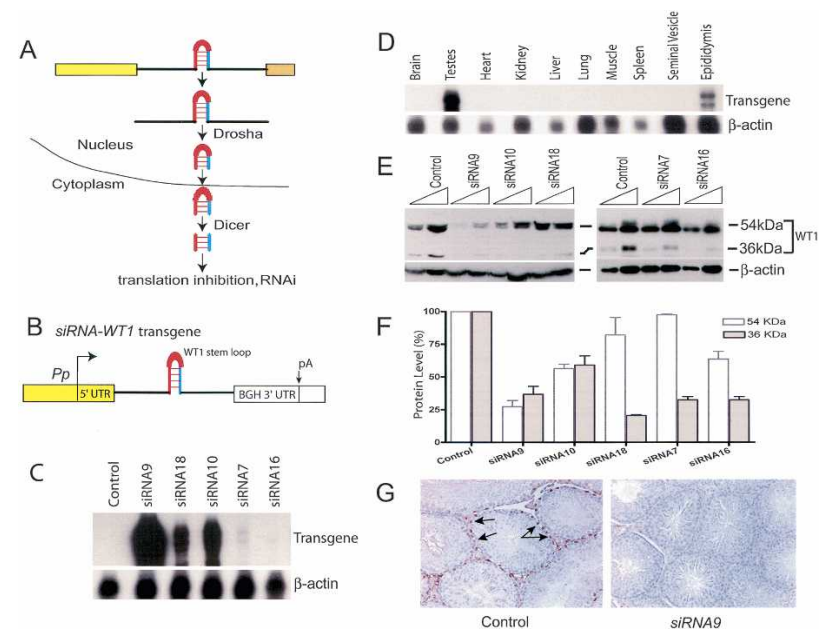

Figure 1. WT1 silencing in SiRNA-WT1 transgenic mice. $(A)$ The canonical microRNA pathway (Tang 2005). (B) The siRNA-WT1 transgene (the WT1 stem-loop sequence is shown in Supplementary Fig. S1A). (UTR) Untranslated region; (BGH) bovine growth hormone; (pA) polyadenylation site. $(C)$ Ribonuclease protection analysis of adult testes RNA $(20 \mu \mathrm{g})$ annealed with transgene- and $\beta$-actinspecific probes. Transgene transcripts were detected with a $3^{\prime}$-UTR probe (Rao et al. 2003). The $\beta$-actin probe served as a loading control. (D) Transgene expression in various tissues from an adult siRNA9 mouse (the same pattern was also observed for the siRNA10 and siRNA16 lines; data not shown). (E) Western blot analysis of the indicated amounts of testicular lysate from control and transgenic mice using a WT1 polyclonal antibody (c19; Santa Cruz, Inc.; 1:1600 dilution). (F) Reduction in WT1 levels in siRNA-WT1 transgenic mice. Expression was quantified by densitometric analysis of three independent Western blots; means \pm SEM. $(G)$ Immunohistochemical analysis of testes from siRNA9 and control littermate adult mice using an anti-mouse WT1 monoclonal antibody (H6; DAKO, Inc.; 1:1000 dilution). Arrows indicate selected control Sertoli cell nuclei that stained positively for WT1. A reduction in WT1 immunostaining was also observed in SiRNA10 mice testes.

We generated seven transgenic mouse lines containing the SiRNA-WT1 transgene, five of which expressed the transgene in their testes, as detected by ribonuclease protection analysis with a probe against the mature mRNA (Fig. 1C). The transgene was detectably expressed in the testes and epididymides and not in any other tissue tested (Fig. 1D). Northern blot analysis with a WT1 short hairpin RNA (shRNA)/intron probe detected $~ 800$ - and -90-nt transcripts, the sizes expected of the transgene pre-RNA and the shRNA processed from it (Supplementary Fig. S1B).

Western blot analysis showed that all five of the SiRNA-WT1 transgenic lines had reduced testicular expression of the $\sim 36-\mathrm{kDa}$ isoform known to be translated from an internal ATG start codon in WT1 (Fig. 1E,F; Scharnhorst et al. 1999). The level of the $\sim 54-\mathrm{kDa}$ isoform, which is translated from the upstream start ATG codon, was significantly reduced in levels in three of the five lines (Fig. 1E,F). In contrast, wild-type levels of WT1 protein were observed in the spleens of siRNA-WT1 mice (Western blot analysis) (data not shown), as expected since the siRNA-WT1 transgene was not expressed in the spleen (Fig. 1D). The reduction in WT1 protein expression in testes was corroborated by immunohistochemical analysis, which showed that WT1 protein levels were reduced in Sertoli cell nuclei from siRNA-WT1 mice (Fig. 1G). We do not know why the isoform translated from the internal ATG was more re- duced in level compared with the upstream ATG-initiated isoform. We speculate that it results from the WT1 siRNA being generated by the miRNA pathway, as this pathway is known to generate inhibitory RNA-protein complexes that act by blocking the initiation phase of translation (Pillai et al. 2005). Consistent with WT1 siRNA acting primarily at the level of translation, WT1 mRNA levels were normal or only slightly reduced in siRNA-WT1 mice testes (Supplementary Fig. S1C).

\section{Germ cell defects in mice with reduced WT1 levels in Sertoli cells}

SiRNA-WT1 transgenic mouse lines exhibiting reduced WT1 expression had numerous male reproductive defects. Five-day timed-mating experiments revealed that the transgenic males were subfertile (control males: 16/ 16 wild-type females impregnated after 5-d cohabitation; SiRNA9 males: 4/15). The transgenic mice had reduced numbers of caudal spermatozoa (Fig. 2A), probably as a result of a testis defect, as they had reduced testis weight (control: $0.230 \pm 0.019 \mathrm{~g}, n=20 ;$ siRNA9: $0.176 \pm 0.017$ g, $n=6 ; P \leq 0.05)$, reduced seminiferous tubule diameter (control: $98.1 \pm 6.1 \mu \mathrm{m}, n=3$; siRNA9: $71.6 \pm 4.8 \mu \mathrm{m}$, $n=3 ; P \leq 0.05)$, and had many more apoptotic spermatogonia and spermatocytes than control mice, as judged using the TUNEL assay (Fig. 2B,C). In addition, the siRNA-WT1 mice had apoptotic post-meiotic germ cells (elongated spermatids), as revealed by electron microscopy (Fig. 2D). This was surprising, as germ cell apoptosis is normally restricted to earlier stages of germ cell development (Maclean et al. 2005); indeed, we never observed apoptotic spermatids in control testes by either electron microscopy or the TUNEL assay. While causing germ cell apoptosis, loss of WT1 did not cause apoptosis or obvious ultrastructural defects in Sertoli cells /data not shown). The transgenic mice also had normal Leydig cells with abundant mitochondria and smooth endoplasmic reticulum (data not shown). To further characterize the testicular defects in the transgenic mice, we per-
A

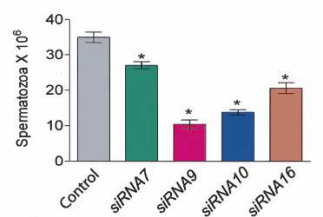

C

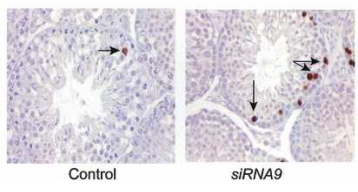

B

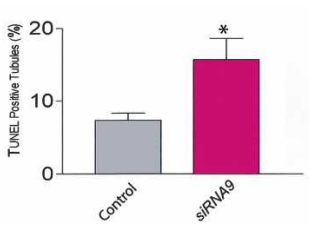

D

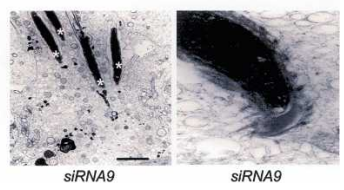

Figure 2. Testes defects in siRNA-WT1 transgenic mice. $(A)$ Caudal spermatozoa counts (means \pm SEM). $\left({ }^{\star}\right) P \leq 0.001 ; n=6$. $(B)$ Quantitative analysis of TUNEL-positive tubules and cells (means \pm SEM). ( $\left.{ }^{\star}\right) P \leq 0.001 ; n=1262$ and 1052 tubules for control and siRNA9, respectively. (C) TUNEL analysis of apoptotic germ cells (arrows) in testes sections. (D) Transmission electron micrographs. The left panel shows a cluster of elongated spermatids deep within the seminiferous epithelium undergoing apoptosis (asterisks), and the right panel shows a high-magnification view of a step14 spermatid with condensed chromatin, characteristic of an early stage of apoptosis. 


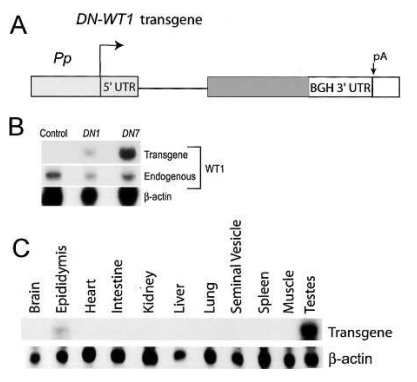

Figure 3. Expression of the DN-WT1 transgene. $(A)$ The $D N-W T 1$ transgene. Solid bar is DN-WT1 coding region; see Figure 1 for definition of abbreviations. $(B)$ RNase protection analysis of adult testes RNA $(20 \mu \mathrm{g})$ from the DN1 and DN7 lines, performed with a WT1 probe that distinguishes between endogenous and transgene transcripts. Transgene transcripts were expressed at $\sim 1.2$-fold and approximately twofold higher levels than endogenous WT1 transcripts in the testes of DN1 and DN7 mice, respectively (when corrected for $\left[{ }^{32} \mathrm{P}\right] \mathrm{UTP}$ content in the protected bands). (C) RNase protection analysis of tissue RNA $(20 \mu \mathrm{g})$ from an adult DN7 mouse annealed with the transgene-specific probe used in Figure 1C.

formed high-magnification light microscopy. This analysis revealed four phenotypic effects: (1) loss of germ cells (consistent with increased germ cell apoptosis), (2) reduced Sertoli cell height, (3) increased frequency of vacuoles in Sertoli cells, and (4) dilation of the tubular lumen (Supplementary Fig. S2).

We predicted that an additional factor besides reduced spermatozoa count was responsible for the hypofertility of the siRNA-WT1 mice, as more dramatic reductions in spermatozoa counts (up to 10 -fold) caused by other genetic perturbations still permit fertility (Kumar et al. 2001). In agreement with this prediction, we found that SiRNA-WT1 mice had a lower proportion of motile spermatozoa compared with control mice (control: $50 \pm 4.5 \%$, $n=8$; siRNA9: $25 \pm 5.5 \%, n=4 ; P<0.005)$. While, in theory, this defect could result from a loss of WT1 expression in the epididymis (as the SiRNA-WT1 transgene is expressed in the epididymis) (Fig. 1D), we believe this is unlikely as we could not detect endogenous WT1 expression in epididymides from wild-type mice by Western blotting, ribonuclease protection, or real-time RTPCR (data not shown). Thus, the defect in the development of motility-competent germ cells is more likely to be due to the loss of WT1 expression in Sertoli cells.

\section{Germ cell defects in mice expressing dominant-negative WT1 in Sertoli cells}

The phenotypic alterations in the siRNA-WT1 mice suggest that WT1 expression in Sertoli cells is essential for both the survival and the proper maturation of germ cells. However, it is possible that the siRNA-WT1 transgene down-regulated the expression of another gene (in addition to WT1) whose loss is responsible for some or all of the phenotypic alterations in SiRNA-WT1 mice. As an independent test of WT1's function in the testis, we generated a transgene encoding a potent dominant-negative form of WT1 expressed from the Pp Sertoli cell promoter (Fig. 3A). This transgene encodes a frameshift allele of WT1 [WT (PM)] that was originally isolated from a patient with Denys-Drash syndrome (Reddy et al. 1995). We chose this particular mutant because even low levels of it are sufficient to inhibit wild-type WT1 function in cultured cells (Reddy et al. 1995).
We obtained two independent transgenic lines, $D N 1$ and DN7, containing the DN-WT1 transgene (Fig. 3B). Both lines expressed the transgene only in testes and epididymides (Fig. 3C; data not shown). In situ hybridization analysis with a transgene-specific probe detected expression in the cytoplasm of Sertoli cells but not in germ or interstitial cells (Supplementary Fig. S3A,B), consistent with our previous studies on $\mathrm{Pp}$ expression (Lindsey and Wilkinson 1996; Sutton et al. 1998; Rao et al. 2003). Sertoli cell-specific expression was confirmed by immunohistochemical analysis (Supplementary Fig. 3C).

The DN-WT1 transgenic mice had essentially the same phenotype as the siRNA-WT1 mice. They were subfertile (Fig. 4A) and had low spermatozoa counts (Fig. 4B). A testis defect was responsible, as they had reduced testis weight (control: $0.24 \pm 0.02 \mathrm{~g}, n=10 ; D N 1$ : $0.19 \pm 0.01 \mathrm{~g}, n=8 ; D N 7: 0.16 \pm 0.01 \mathrm{~g}, n=7 ; P \leq 0.05)$, reduced tubule diameter (control: $98.0 \pm 6.1 \mu \mathrm{m}, n=3$; DN1: $81.2 \pm 3.0 \mu \mathrm{m}, n=3$; DN7: $72.5 \pm 3.5 \mu \mathrm{m}, n=3$; $P \leq 0.05)$, and a marked increase in germ cell apoptosis (Fig. 4C). Apoptosis was increased in the seminiferous epithelial stages that normally have apoptotic germ cells (stages I-IV and XII) (MacLean et al. 2005), as well as stages that normally do not (stages VII-XI) (Fig. 4D). This increased germ cell apoptosis explains why DN1 and DN7 also had reduced numbers of spermatids in their
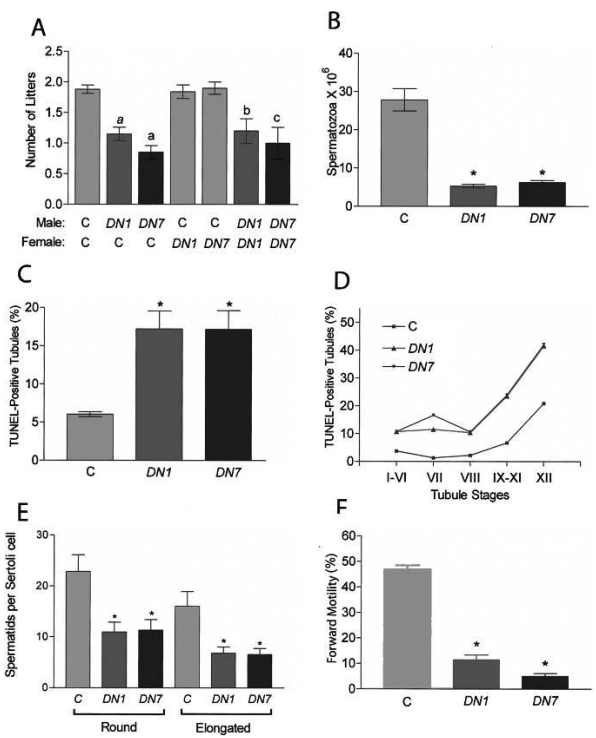

Figure 4. Testes defects in DN-WT1 transgenic mice. (A) Mean number of litters obtained from 60 -d pairings of $D N 1$ and $D N 7$ mice and control littermates (labeled "C"). (a) $P \leq 0.001$; (b) $P<0.05$; (c) $P \leq 0.01 ; n=25,20$, and 20 for control, $D N 1$, and $D N 7$, respectively. After a 5 -d cohabitation, only $20 \%$ of DN1 males sired litters $(n=25)$ (data not shown), similar to the percentage for siRNA9 mice (27\%; see text). (B) Caudal spermatozoa counts. $\left.{ }^{\star}\right) P \leq 0.001 ; n=8$ mice per group. $(C)$ Percentage of TUNEL-positive tubules. $\left({ }^{\star}\right)$ $P \leq 0.001 ; n=628,830$, and 770 tubules for $D N 1, D N 7$, and control mice, respectively. $(D)$ Percentage of TUNEL-positive tubules at different stages of the seminiferous epithelial cycle. In addition to having apoptotic spermatogonia and mitotic spermatocytes, both of which normally undergo apoptosis, the DN-WT1 mice had apoptotic round spermatids (data not shown). (E) Number of round and elongated spermatids in stage VII tubules. $\left({ }^{\star}\right) P \leq 0.05 ; n=6$ mice per group. $(F)$ Percentage of sperm displaying forward motility. $\left.{ }^{\star}\right)$ $P \leq 0.001 ; n=10$ mice per group. The data in panels $A-C, E$, and $F$ are means \pm SEM. 
seminiferous tubules (Fig. 4E) and sonication-resistant (differentiated) spermatids (control: $13.57 \pm 2.14 \times 10^{6}$, $n=8 ; D N 1: 7.48 \pm 1.71 \times 10^{6}, n=8 ; D N 7: 7.10 \pm 0.91 \times 10^{6}$, $n=8 ; P \leq 0.05)$. Lastly, the $D N-W T 1$ mice had fewer forward-motile spermatozoa compared with control mice (Fig. 4F). Because the DN-WT1 mice had the same set of phenotypic alterations as the siRNA-WT1 mice, we believe this unambiguously demonstrates that WT1 expression in Sertoli cells is essential to promote the survival of the adjacent germ cells and their maturation into motile sperm.

\section{WT1-regulated genes in Sertoli cells}

To identify WT1-regulated genes that may cause or contribute to these phenotypic effects, we performed microarray analysis on testes from $D N-W T 1$ and control adult mice. Interestingly, many of the genes whose expression was altered by the DN-WT1 transgene encode signaling, energy-related, and structural proteins that are good candidates to mediate the biological functions of WT1 in Sertoli cells (Supplementary Table S1).

We chose three of these genes, epidermal growth factor receptor pathway substrate-8 (Eps8), Icap1- $\alpha$ (bodenin), and importin- $\alpha 1$, for further analysis because of their functional attributes (discussed below) and their high expression in testes (Faisst and Gruss 1998; data not shown). Ribonuclease protection analysis confirmed that all three of these genes exhibited altered expression in DN-WT1 mice (Supplementary Fig. S4A-C). They exhibited an identical expression pattern in SiRNA-WT1 testes (Fig. 5A), providing strong evidence that all three are bona fide targets of WT1.

Two lines of evidence indicated that the two genes up-regulated by WT1 (Eps8 and importin- $\alpha 1$ ) are regulated in a cell-autonomous manner in Sertoli cells. First, the transcripts from both genes were highly enriched in the Sertoli cell fraction purified from adult mouse testes (Supplementary Table S2). Second, both were positively regulated by WT1 in the Sertoli cell line 15P-1, as forced expression of WT1 increased endogenous Eps8 and importin- $\alpha 1$ mRNA levels, while transfection of a $D N-W T 1$ vector or a siRNA-WT1 vector had the opposite effect (Supplementary Fig. S4D,E).

Eps8 and Icap 1- $\alpha$ are crucial signaling molecules that converge to regulate actin-mediated cytoskeletal events (Scita et al. 1999; Degani et al. 2002). Both are $\beta 1$-integrin-binding proteins that regulate cell adhesion and motility in fibroblasts (Bouvard et al. 2003; Calderwood et al. 2003; Innocenti et al. 2003; Disanza et al. 2004). In the testis, $\beta 1$-integrin resides in the ectoplasmic specialization (ES), a testis-specific actin-based adherens junction that continuously disassembles and reassembles to facilitate the migration of developing germ cells across the surface of Sertoli cells (Mruk and Cheng 2004; Siu et al. 2005). Our discovery that WT1 regulates the expression of Eps8 and Icap1- $\alpha$ led us to hypothesize that WT1 controls the formation of the ES. To test this hypothesis, we examined Sertoli cell-germ cell junctions in our WT1deficient transgenic mice by electron microscopy. This analysis revealed that the ES was either disrupted or completely absent in testes from both the siRNA-WT1 and DN-WT1 mice (Fig. 5B-D). Analysis of 300 micrographs of mice testes from SiRNA-WT1, DN-WT1, and littermate control mice (100 micrographs from two mice from each group) showed that $\sim 40 \%$ of spermatids from
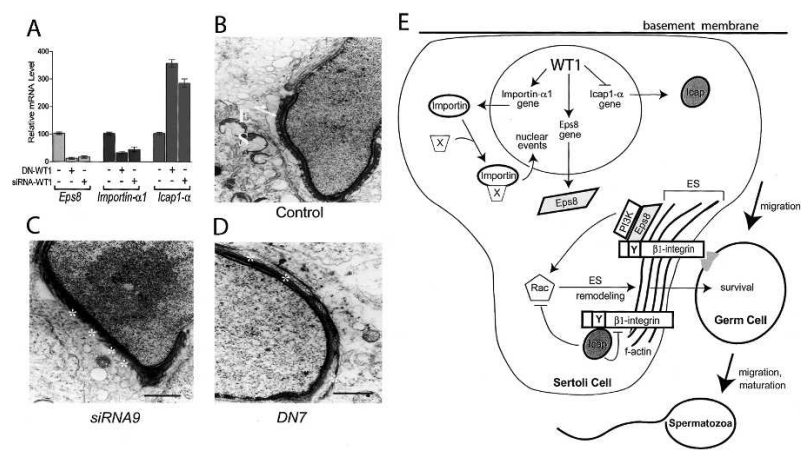

Figure 5. Altered gene expression and disrupted adherens junctions in WT1-deficient mice. (A) The Eps8, Icap1- $\alpha$, and importin- $\alpha 1$ genes are regulated in the same manner in both siRNA-WT1 and $D N$-WT1 mice testes. Real-time RT-PCR analysis was used to determine transcript expression relative to that of L19 mRNA (encodes a ubiquitously expressed ribosomal protein). $(B-D)$ Transmission electron micrographs of testes from siRNA9 (siRNA-WT1), DN7 (DN-WT1), and control littermate mice. The control panel shows a normal step-10 spermatid embedded in a Sertoli cell; the ES junctional complex is present (arrows), and the acrosome extends over the apex and the dorsal curvature of the head. In contrast, the spermatid-Sertoli cell junctions in siRNA-WT1 and DN-WT1 mice often lacked the ES (asterisks). (E) Model of WT1 action in Sertoli cells. Eps8 is a signaling molecule that forms a molecular complex with Rac, phosphoinositide 3-kinase, and $\beta_{1}$-integrin (see text). Because this complex is known to elicit cytoskeletal remodeling, we posit that by up-regulating Eps8, WT1 activates the actin remodeling events at the Sertoli cell membrane essential for migration of germ cells across its surface, as well as for germ cell survival and maturation. We also posit that these remodeling events are enhanced by WT1's ability to also inhibit the expression of Icap $1-\alpha$, which is a negative regulator of Rac activation and $\beta_{1}$-integrin avidity (see text). Lastly, WT1 increases the level of importin- $\alpha 1$, which we propose is necessary for the efficient nuclear import of proteins (designated by " $X "$ ") that control nuclear events essential for spermatogenesis.

the transgenic mouse testes had this ES defect, whereas it was never observed in littermate control mice.

\section{Transcription factors, gonads, and spermatogenesis}

Our study demonstrates that WT1 expression in Sertoli cells is crucial for the survival and development of the adjacent germ cells. This postnatal and adult role is distinct from its previously defined embryonic role promoting the development of the gonad (Little et al. 1999; Park and Jameson 2005). To date, no other transcription factor essential for gonad formation has been definitively shown to later function in spermatogenesis. Other transcription factors that might have such a bifunctional role are Sox8, Sox9, Dax1, and GATA4, all of which control sex determination in the embryonic gonad and continue to be expressed in adult Sertoli cells (Tamai et al. 1996; Ketola et al. 1999; Shen and Ingraham 2002; Park and Jameson 2005).

\section{Model}

Our electron microscopic analyses of WT1-deficient mice provided evidence that WT1 is essential for the formation and normal function of the ES, a specialized junction complex that forms between Sertoli cells and germ cells. To our knowledge, WT1 is the first transcription factor shown to have this ability. We identified 
WT1-regulated signaling molecules that may control ES formation or function. One of these molecules, Eps8, is part of a large complex that also contains the p85 regulatory subunit of phosphoinositide 3-kinase (PI3K), that activates the small G-protein Rac (Innocenti et al. 2003). In fibroblasts, this Eps8-containing complex stimulates actin-mediated cytoskeletal remodeling, cell motility, and cell adhesion (Scita et al. 1999). Eps8 has an intimate role in this cytoskeletal remodeling, as it directly binds to F-actin (Disanza et al. 2004). We propose that this Eps8-PI3K-Rac pathway also operates in the testis by directing the orderly migration of differentiating germ cells across the surface of the Sertoli cell (Fig. 5E). In support of this model, PI3K is essential for fertility in male mice (Blume-Jensen et al. 2000), and Rac is present at the interface of rat Sertoli cells and spermatids (Chapin et al. 2001) and has a conserved role in male fertility that extends to at least Drosophila melanogaster (Raymond et al. 2004).

Reinforcing this mechanism is WT1's ability to inhibit the expression of Icap $1-\alpha$, a negative regulator of Rac1/ Cdc42-mediated signaling and $\beta_{1}$-integrin avidity (Degani et al. 2002; Bouvard et al. 2003). Like Eps8, Icap1- $\alpha$ has a phosphotyrosine-binding domain that binds to tyrosine-containing motifs in the cytoplasmic tail of $\beta_{1}$ integrin (Chang et al. 2002; Calderwood et al. 2003). This suggests that Icap1- $\alpha$ and Eps8 compete for binding to $\beta_{1}$-integrin and that the outcome of this competition dictates whether integrin-mediated cytoskeletal remodeling occurs or not. By both reducing the expression of the negative regulator Icap $1-\alpha$ and stimulating the expression of the positive regulator Eps8, we posit that WT1 strongly swings the balance toward cytoskeletal remodeling (Fig. 5E). We further propose that this remodeling is necessary for the normal survival and maturation of germ cells. Consistent with this, we found that WT1deficient Sertoli cells are less able to support the survival of germ cells and their maturation into motile spermatozoa.

The third WT1-regulated molecule that we identified in the testis is importin- $\alpha 1$, an adaptor that mediates the import of a subset of proteins into the nucleus. Although importin- $\alpha 1$ 's role in mammalian fertility is not known, importin- $\alpha$ molecules are essential for male fertility in both D. melanogaster and Caenorhabditis elegans (Hogarth et al. 2005). By augmenting importin- $\alpha 1$ levels in Sertoli cells, we propose that WT1 stimulates the nuclear import of key molecules that promote mammalian spermatogenesis. Several testicular regulatory proteins, including Sry, Sox9, CREM, CREB, Smads, and cyclins, display altered nuclear-cytoplasmic distributions in response to developmental cues or mutation (Hogarth et al. 2005).

\section{Tissue-specific RNAi}

Our study introduces a new in vivo RNAi approach that we believe will be generally applicable to elucidating the cell-type-specific function of genes. Complementing our approach is another recently developed RNAi method that involves the use of a Pol III promoter-driven siRNA whose expression is regulated in a tissue-specific manner by a Pol II-driven Cre recombinase (Ventura et al. 2004). While potentially powerful, this Cre-based method requires the introduction of two separate transgenes into embryonic stem cells, followed by generation of chi- meric mice. In contrast, our approach is relatively simple, requiring that only one transgene be introduced into mice by standard transgenic technology. By substituting different tissue-specific or regulated promoters and stem-loops corresponding to different gene targets, this system has the potential to knock down the expression of virtually any gene in a cell-type-specific and temporally regulated manner.

\section{Materials and methods}

\section{Plasmids}

The DN-WT1 (Pem-236) transgene construct consists of the pCITE-2A (Clontech) plasmid containing the $P p$ Sertoli cell promoter, the TCR- $\beta$ $\mathrm{C} \beta 2.1$ intron, the WT (PM) cDNA, and the bovine growth hormone 3 'untranslated region and polyadenylation signal (bGH-pA). Pp 5'-flanking sequences (-655 to -1 with respect to the Rhox 5 start ATG codon in exon 3) were amplified from the plasmid Pem-121 (Wayne et al. 2002) with primers containing NcoI and NdeI restriction sites. The TCR- $\beta$ C $\beta 2.1$ intron was amplified from the $\beta-290$ vector (Gudikote and Wilkinson 2002) and cloned into the NdeI and EcoRI restriction sites of pCITE-2A. The WT (PM) cDNA was digested from the RSV-WT (PM) vector (Reddy et al. 1995) and subcloned into the EcoRI and SalI sites of pCITE-2A. The bGH-pA sequences were amplified from the plasmid Pem-121 (Wayne et al. 2002) and inserted into the SalI and NotI sites downstream of the WT (PM) cDNA in pCITE-2A. The siRNA-WT1 construct (Pem-260) is identical to the DN-WT1 construct except that it lacks the WT (PM) cDNA and instead has a hairpin loop (Supplementary Fig. 1A), which contains sequences shown previously to elicit WT1 RNAi (Davies et al. 2004), inserted into the TCR- $\beta$ C $\beta 2.1$ intron at its MunI site.

\section{Animals}

All experiments were performed in accordance with National Institutes of Health guidelines for care and use of animals. Mice were kept on a 12-h light/12-h dark cycle; all experiments were performed on mice between 16 and 18 wk old. The DN-WT1 and siRNA-WT1 transgenic mice (B6D2F1 strain) were made by liberating the transgene from the plasmid backbone by digestion with PmeI and XhoI and then injecting this DNA into mouse pronuclei. Two DN-WT1 (DN1 and DN7) and seven siRNAWT1 (siRNA9, siRNA10, siRNA15, siRNA16, siRNA18, siRNA35, and siRNA36) founder transgenic lines were generated, as detected by PCR using tail DNA as a template. Tissue preparation, spermatozoa and spermatid counts, the TUNEL and spermatozoa motility assays, and Sertoli and interstitial cell-fraction enrichment were done as described previously (MacLean et al. 2005). The level of significance for all data was analyzed using the Student's $t$-test.

\section{RNA and protein analysis}

Total cellular RNA was isolated as described (Lindsey and Wilkinson 1996). RNase protection and real-time RT-PCR analyses were done as described previously (Rao et al. 2003; MacLean et al. 2005, respectively). Histological, immunohistochemical, and Western blot analyses were performed as described previously (Rao et al. 2003).

\section{Acknowledgments}

We thank J. Licht (Mount Sinai School of Medicine, New York) for providing the RSV-WT (PM) construct and Wai-kin Chen (M.D. Anderson Cancer Center, Houston) for providing the U6-siRNA-luc construct.

\section{References}

Armstrong, J.F., Pritchard-Jones, K., Bickmore, W.A., Hastie, N.D., and Bard, J.B. 1993. The expression of the Wilms' tumour gene, WT1, in the developing mammalian embryo. Mech. Dev. 40: 85-97.

Blume-Jensen, P., Jiang, G., Hyman, R., Lee, K.F., O'Gorman, S., and Hunter, T. 2000. Kit/stem cell factor receptor-induced activation of phosphatidylinositol 3'-kinase is essential for male fertility. Nat. Genet. 24: 157-162.

Bouvard, D., Vignoud, L., Dupe-Manet, S., Abed, N., Fournier, H.N., Vincent-Monegat, C., Retta, S.F., Fassler, R., and Block, M.R. 2003. Dis- 
ruption of focal adhesions by integrin cytoplasmic domain-associated protein-1 $\alpha$. J. Biol. Chem. 278: 6567-6574.

Calderwood, D.A., Fujioka, Y., de Pereda, J.M., Garcia-Alvarez, B., Nakamoto, T., Margolis, B., McGlade, C.J., Liddington, R.C., and Ginsberg, M.H. 2003. Integrin $\beta$ cytoplasmic domain interactions with phosphotyrosine-binding domains: A structural prototype for diversity in integrin signaling. Proc. Nat1. Acad. Sci. 100: 2272-2277.

Chang, D.D., Hoang, B.Q., Liu, J., and Springer, T.A. 2002. Molecular basis for interaction between Icap $1 \alpha$ PTB domain and $\beta 1$ integrin. J. Biol. Chem. 277: 8140-8145.

Chapin, R.E., Wine, R.N., Harris, M.W., Borchers, C.H., and Haseman, J.K. 2001. Structure and control of a cell-cell adhesion complex associated with spermiation in rat seminiferous epithelium. J. Androl. 22: 1030-1052.

Davies, J.A., Ladomery, M., Hohenstein, P., Michael, L., Shafe, A., Spraggon, L., and Hastie, N. 2004. Development of an siRNA-based method for repressing specific genes in renal organ culture and its use to show that the Wt1 tumour suppressor is required for nephron differentiation. Hum. Mol. Genet. 13: 235-246.

Degani, S., Balzac, F., Brancaccio, M., Guazzone, S., Retta, S.F., Silengo, L., Eva, A., and Tarone, G. 2002. The integrin cytoplasmic domainassociated protein ICAP-1 binds and regulates Rho family GTPases during cell spreading. J. Cell Biol. 156: 377-387.

Disanza, A., Carlier, M.F., Stradal, T.E., Didry, D., Frittoli, E., Confalonieri, S., Croce, A., Wehland, J., Di Fiore, P.P., and Scita, G. 2004. Eps8 controls actin-based motility by capping the barbed ends of actin filaments. Nat. Cell Biol. 6: 1180-1188.

Faisst, A.M. and Gruss, P. 1998. Bodenin: A novel murine gene expressed in restricted areas of the brain. Dev. Dyn. 212: 293-303.

Fedoriw, A.M., Stein, P., Svoboda, P., Schultz, R.M., and Bartolomei, M.S. 2004. Transgenic RNAi reveals essential function for CTCF in H19 gene imprinting. Science 303: 238-240.

Gudikote, J.P. and Wilkinson, M.F. 2002. T-cell receptor sequences that elicit strong down-regulation of premature termination codon-bearing transcripts. EMBO J. 21: 125-134.

Hogarth, C., Itman, C., Jans, D.A., and Loveland, K.L. 2005. Regulated nucleocytoplasmic transport in spermatogenesis: A driver of cellular differentiation? Bioessays 27: 1011-1025.

Innocenti, M., Frittoli, E., Ponzanelli, I., Falck, J.R., Brachmann, S.M., Di Fiore, P.P., and Scita, G. 2003. Phosphoinositide 3-kinase activates Rac by entering in a complex with Eps8, Abi1, and Sos-1. J. Cell Biol. 160: $17-23$.

Ketola, I., Rahman, N., Toppari, J., Bielinska, M., Porter-Tinge, S.B., Tapanainen, J.S., Huhtaniemi, I.T., Wilson, D.B., and Heikinheimo, M. 1999. Expression and regulation of transcription factors GATA-4 and GATA-6 in developing mouse testis. Endocrinology 140: 14701480.

Kumar, T.R., Varani, S., Wreford, N.G., Telfer, N.M., de Kretser, D.M., and Matzuk, M.M. 2001. Male reproductive phenotypes in double mutant mice lacking both FSH $\beta$ and activin receptor IIA. Endocrinology 142: 3512-3518.

Lindsey, J.S. and Wilkinson, M.F. 1996. Pem: A testosterone- and LHregulated homeobox gene expressed in mouse Sertoli cells and epididymis. Dev. Biol. 179: 471-484.

Little, M., Holmes, G., and Walsh, P. 1999. WT1: What has the last decade told us? Bioessays 21: 191-202.

MacLean II, J.A., Chen, M.A., Wayne, C.M., Bruce, S.R., Rao, M., Meistrich, M.L., Macleod, C., and Wilkinson, M.F. 2005. Rhox: A new homeobox gene cluster. Cell 120: 369-382.

Mruk, D.D. and Cheng, C.Y. 2004. Cell-cell interactions at the ectoplasmic specialization in the testis. Trends Endocrinol. Metab. 15: 439447.

Park, S.Y. and Jameson, J.L. 2005. Minireview: Transcriptional regulation of gonadal development and differentiation. Endocrinology 146: 1035-1042.

Pelletier, J., Schalling, M., Buckler, A.J., Rogers, A., Haber, D.A., and Housman, D. 1991. Expression of the Wilms' tumor gene WT1 in the murine urogenital system. Genes \& Dev. 5: 1345-1356.

Pillai, R.S., Bhattacharyya, S.N., Artus, C.G., Zoller, T., Cougot, N., Basyuk, E., Bertrand, E., and Filipowicz, W. 2005. Inhibition of translational initiation by Let-7 MicroRNA in human cells. Science 309: 1573-1576.

Rao, M.K., Wayne, C.M., and Wilkinson, M.F. 2002. Pem homeobox gene regulatory sequences that direct androgen-dependent developmentally regulated gene expression in different subregions of the epididymis. J. Biol. Chem. 277: 48771-48778.

Rao, M.K., Wayne, C.M., Meistrich, M.L., and Wilkinson, M.F. 2003. Pem homeobox gene promoter sequences that direct transcription in a Sertoli cell-specific, stage-specific, and androgen-dependent manner in the testis in vivo. Mol. Endocrinol. 17: 223-233.

Raymond, K., Bergeret, E., Avet-Rochex, A., Griffin-Shea, R., and Fauvarque, M.O. 2004. A screen for modifiers of RacGAP(84C) gain-offunction in the Drosophila eye revealed the LIM kinase Cdi/TESK1 as a downstream effector of Rac1 during spermatogenesis. J. Cell Sci. 117: 2777-2789.

Reddy, J.C., Morris, J.C., Wang, J., English, M.A., Haber, D.A., Shi, Y., and Licht, J.D. 1995. WT1-mediated transcriptional activation is inhibited by dominant negative mutant proteins. J. Biol. Chem. 270: 10878-10884.

Scharnhorst, V., Dekker, P., van der Eb, A.J., and Jochemsen, A.G. 1999. Internal translation initiation generates novel WT1 protein isoforms with distinct biological properties. J. Biol. Chem. 274: 23456-23462.

Scita, G., Nordstrom, J., Carbone, R., Tenca, P., Giardina, G., Gutkind, S., Bjarnegard, M., Betsholtz, C., and Di Fiore, P.P. 1999. EPS8 and E3B1 transduce signals from Ras to Rac. Nature 401: 290-293.

Shen, J.H. and Ingraham, H.A. 2002. Regulation of the orphan nuclear receptor steroidogenic factor 1 by Sox proteins. Mol. Endocrinol. 16: 529-540.

Siu, M.K.Y., Wong, C.H., Lee, W.M., and Cheng, C.Y. 2005. Sertoli-germ cell anchoring junction dynamics in the testis are regulated by an interplay of lipid and protein kinases. J. Biol. Chem. 280: 2502925047.

Sutton, K.A., Maiti, S., Tribley, W.A., Lindsey, J.S., Meistrich, M.L., Bucana, C.D., Sanborn, B.M., Joseph, D.R., Griswold, M.D., Cornwall, G.A., et al. 1998. Androgen regulation of the Pem homeodomain gene in mice and rat Sertoli and epididymal cells. J. Androl. 19: 21-30.

Tamai, K.T., Monaco, L., Alastalo, T.P., Lalli, E., Parvinen, M., and Sassone-Corsi, P. 1996. Hormonal and developmental regulation of DAX-1 expression in Sertoli cells. Mol. Endocrinol. 10: 1561-1569.

Tang, G. 2005. siRNA and miRNA: An insight into RISCs. Trends Biochem. Sci. 30: 106-114.

Ventura, A., Meissner, A., Dillon, C.P., McManus, M., Sharp, P.A., Van Parijs, L., Jaenisch, R., and Jacks, T. 2004. Cre-lox-regulated conditional RNA interference from transgenes. Proc. Natl. Acad. Sci. 101: 10380-10385.

Wayne, C.M., Sutton, K., and Wilkinson, M.F. 2002. Expression of the Pem homeobox gene in Sertoli cells increases the frequency of adjacent germ cells with deoxyribonucleic acid strand breaks. Endocrinology 143: 4875-4885.

Zeng, Y. and Cullen, B.R. 2004. Structural requirements for pre-microRNA binding and nuclear export by Exportin 5. Nucleic Acids Res. 32: 4776-4785. 


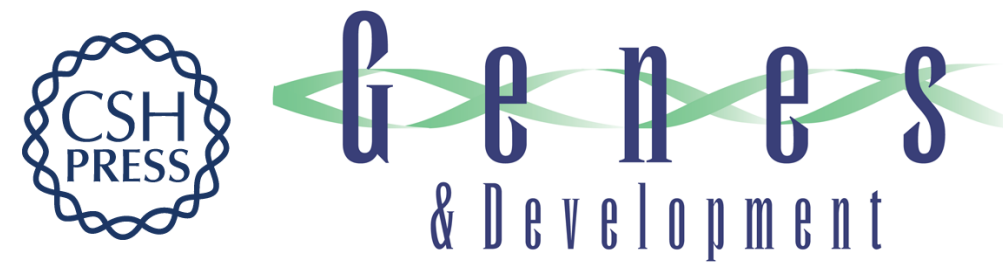

\section{Tissue-specific RNAi reveals that WT1 expression in nurse cells controls germ cell survival and spermatogenesis}

Manjeet K. Rao, John Pham, J. Saadi Imam, et al.

Genes Dev. 2006, 20:

Access the most recent version at doi:10.1101/gad1367806

Supplemental http://genesdev.cshlp.org/content/suppl/2005/12/29/20.2.147.DC1
Material

References This article cites 37 articles, 14 of which can be accessed free at: http://genesdev.cshlp.org/content/20/2/147.full.html\#ref-list-1

License

Email Alerting Receive free email alerts when new articles cite this article - sign up in the box at the top Service right corner of the article or click here.

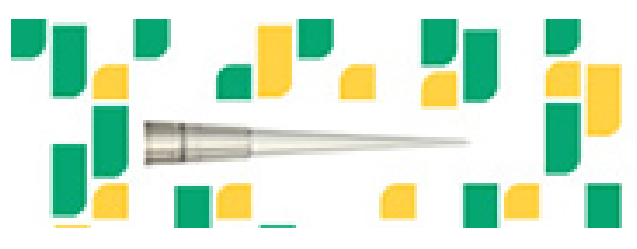

Focused on your science. 